\title{
OPEN ARCH: integrated care at the primary-secondary interface for the community-dwelling older person with complex needs
}

\author{
Jennifer Mann ${ }^{\mathrm{A}, \mathrm{B}, \mathrm{D}}$, Rachel Quigley ${ }^{\mathrm{A}, \mathrm{B}}$, Desley Harvey ${ }^{\mathrm{A}, \mathrm{C}}$, Megan Tait ${ }^{\mathrm{A}}$, \\ Gillian Williams ${ }^{\mathrm{A}}$ and Edward Strivens ${ }^{\mathrm{A}, \mathrm{B}}$

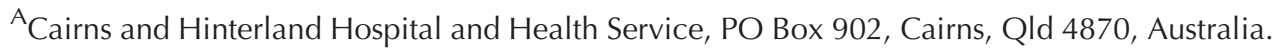 \\ ${ }^{B}$ College of Public Health, Medicine and Veterinary Sciences, James Cook University, PO Box 6811, \\ Cairns, Qld 4870, Australia. \\ ${ }^{C}$ College of Healthcare Sciences, James Cook University, PO Box 6811, Cairns, QId 4870, Australia. \\ ${ }^{D}$ Corresponding author. Email: jennifer.mann3@health.qld.gov.au
}

\begin{abstract}
Optimal care of community-dwelling older Australians with complex needs is a national imperative. Suboptimal care that is reactive, episodic and fragmented, is costly to the health system, can be life threatening to the older person and produces unsustainable carer demands. Health outcomes would be improved if services (health and social) are aligned towards community-based, comprehensive and preventative care. Integrated care is person-focussed in outlook and defies a condition-centric approach to healthcare delivery. Integration is a means to support primary care, with the volume and complexity of patient needs arising from an ageing population. Older Persons Enablement and Rehabilitation for Complex Health Conditions (OPEN ARCH) is a targeted model of care that improves access to specialist assessment and comprehensive care for older persons at risk of functional decline, hospitalisation or institutionalised care. OPEN ARCH was developed with primary care as the central integrating function and is built on four values of quality care: preventative health care provided closer to home; alignment of specialist and generalist care; care coordination and enablement; and primary care capacity building. Through vertical integration at the primarysecondary interface, OPEN ARCH cannot only improve the quality of care for clients, but improves the capacity of primary care to meet the needs of this population.
\end{abstract}

Additional keywords: case management, delivery of health care: integrated, organisation: models, primary health care.

Received 24 September 2019, accepted 19 February 2020, published online 15 April 2020

\section{Introduction}

Optimal care of community-dwelling older Australians with complex needs is a national imperative. Suboptimal care that is reactive, episodic and fragmented, is costly to the health system, can be life-threatening to the older person and produces unsustainable carer demands (AIHW 2014; Swierssen and Duckett 2016; De Carvalho et al. 2017; Harvey et al. 2017). As the population ages and multi-morbidity becomes the norm, older persons with complex needs place excessive pressure on the GP as gatekeeper to specialist medical, community and social supports (Bird et al. 2007; Tieman et al. 2007; Katterl et al. 2012; McPake and Mahal 2017).

Personal complexity arises from the interface between clinical and bio-psycho-social instability and has a negative effect on function, independence and quality of life (Agency for Clinical Innovation 2014). The community-dwelling older person with complex needs may have multiple medical diagnoses; conditions of frailty or geriatric syndromes; may experience an unstable living environment; and their informal caring arrangement may be unsustainable. These persons often require assistance from multiple medical healthcare professionals, have significantly longer hospital stays and are likely to require formal supports to continue living at home (Bird et al. 2007; Beswick et al. 2008; Agency for Clinical Innovation 2014).

In Australia, disconnection of health and social care and malalignment of acute, primary and community services compromise the provision of comprehensive, patient-focussed care (Productivity Commission 2017). System complexity places the community-dwelling older person in a vulnerable situation. To avoid rapid functional decline, hospitalisation and institutionalisation, the older person with complex needs must successfully master multiple complex systems.

Health outcomes would be improved if services (health and social) were aligned towards community-based, comprehensive and preventative care (Beswick et al. 2008). Integration is the contemporary solution (Curry and Ham 2010; Goodwin 2013; 


\section{What is known about the topic?}

- Optimal care of community-dwelling older Australians with complex needs is a national imperative, and health outcomes would be improved if services were aligned towards community-based, comprehensive and preventative care.

\section{What does this paper add?}

- Through vertical integration at the primary-secondary interface, OPEN ARCH improves the quality of care for clients and increases the capacity of primary care to meet the needs of this population.

Wodchis et al. 2015; World Health Organization 2016). Integrated care is '...the search to connect the healthcare system (acute, primary medical and skilled) with other human service systems (e.g. long-term care, education, vocational and housing services) in order to improve outcomes (clinical, satisfaction and efficiency)' (Leutz 1999, pp. 77-78). Integrated care is personfocussed in outlook and defies a condition-centric approach to healthcare delivery. Integration is a means to support primary care with the volume and complexity of patient needs arising from an ageing population (Valentijn et al. 2013; Mitchell et al. 2015).

Integrated care is having a renaissance in Australian policy. At the national level, My Health Record is a nation-wide solution for the integration of medical records, and My Healthcare Home is a primary care-based strategy that shifts the focus of consultation from condition-based to comprehensive patient-focussed care (Australian Digital Health Agency 2018; Australian Government Department of Health 2019a). State Governments are following suit. The Queensland Department of Health released the Integrated Care Innovation Fund (ICIF) in 2016, with the goal of piloting system-level integration between Queensland Health Hospital and Health Services and regional Primary Health Networks (State of Queensland 2019). The purpose of this article is to describe a unique program of integrated care for communitydwelling older persons that was co-funded by ICIF and the North Queensland Primary Health Network (NQPHN). Ethical approval was granted by the Far North Queensland Human Research Ethics Committee (HREC/17/QCH/104 - 1174).

\section{Setting}

The Cairns and Hinterland Hospital and Health Service (CHHHS) is located in Far North Queensland, Australia. The CHHHS is characterised by a higher rate of socioeconomic disadvantage compared with the rest of Queensland, with almost $60 \%$ of the population in the bottom two quintiles of disadvantage (Queensland Government 2018). The CHHHS has the largest absolute Aboriginal and Torres Strait Islander population of any health service in Queensland, with 14\% of the population identifying as Aboriginal or Torres Strait Islander compared with 4\% across the State (Queensland Government 2018). Approximately $20 \%$ of the CHHHS population were over the age of 60 years in 2015 , with this age group projected to grow the fastest of any in this region over the next 10 years (Queensland Government 2018).

\section{Designing a new model of care}

In 2013, the Australian Primary Health Care Research Institute funded a study to examine care transitions of communitydwelling older persons across acute, sub-acute and primary care within the CHHHS, to assist in deriving a whole-of-system approach to optimal patient care (Harvey et al. 2017). A need to embed early intervention and prevention through improved screening, assessment, care co-ordination and formalised inreach and out-reach linkages was highlighted (Harvey et al. 2017). These recommendations formed the basis for the Older Persons Enablement and Rehabilitation for Complex Health Conditions (OPEN ARCH).

OPEN ARCH is an Australian-first model of care for the community-dwelling older person with complex needs, established in 2017 to improve access to specialist assessment and comprehensive care for older persons at risk of functional decline, hospitalisation or institutionalised care. The CHHHS received AU\$1.2M funding from the ICIF and AU\$400 000 from the NQPHN over a 2-year period to develop, implement and evaluate OPEN ARCH within the Cairns region.

OPEN ARCH harnesses the integrative functions of primary care (first contact, continuous, comprehensive and coordinated care) to create integration at the person (micro) and system (meso) levels (Valentijn et al. 2013). OPEN ARCH does not duplicate existing health or social services, but identifies health concerns early in the trajectory of the person's illness, centralises a plan of care and coordinates supports tailored to individualised needs.

\section{The model}

OPEN ARCH provides care to GP-referred, communitydwelling, non-Aboriginal and Torres Strait Islanders aged $>70$ years, and Aboriginal and Torres Strait Islander persons aged $>50$ years.

Each client of OPEN ARCH is assigned an Enablement Officer (EO) (allied health or nursing), and a geriatrician. The OPEN ARCH service flow is characterised by five stages (Fig. 1): client identification and referral; Comprehensive Geriatric Assessment (CGA); person-directed care planning; coordination of supports; and transfer of care.

OPEN ARCH has developed a culturally safe model to promote service access for Aboriginal and Torres Strait Islander clients. Flexibility in service flow, utilisation of the Aboriginal and Torres Strait Islander Health Worker in assessment and coordination, and a patient-directed pace of program delivery support engagement of Aboriginal and Torres Strait Islander clients (Smith et al. 2011; Davy et al. 2016).

The OPEN ARCH intervention is built on four values of quality integrated care: preventative health care provided closer to home; alignment of specialist/generalist care; care coordination and enablement; and primary care capacity building. These four values are described below with case examples.

\section{Preventative health care provided closer to home}

OPEN ARCH receives referrals directly from the treating GP, for those older clients identified through their care as complex and at-risk of functional decline or hospitalisation. The service goes to where the client is; that is, to the person's home or the GP 


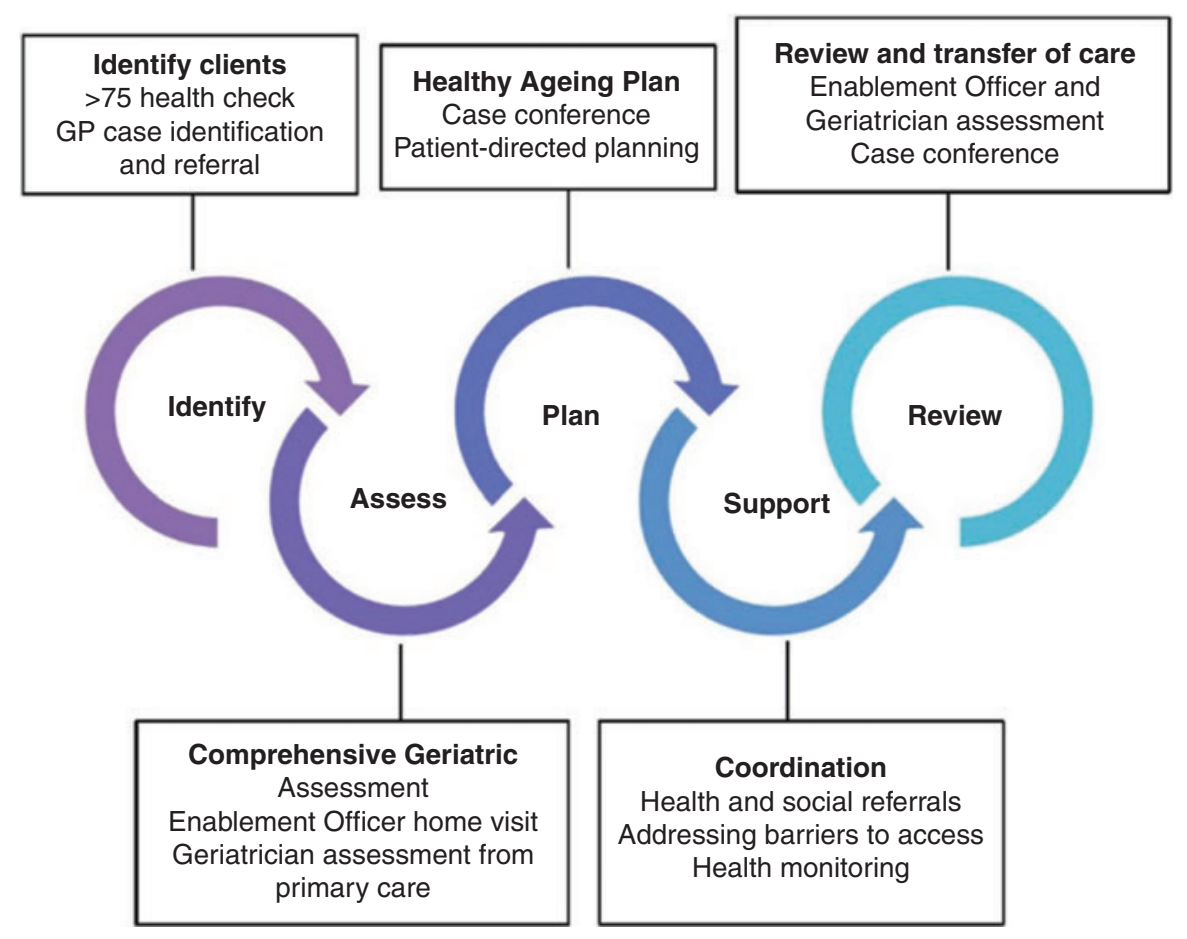

Fig. 1. Service flow of the Older Persons Enablement and Rehabilitation for Complex Health Conditions (OPEN ARCH) program.

clinic. OPEN ARCH provides comprehensive assessment within the home environment to facilitate depth of understanding of personal circumstances and the creation of a personalised care plan.

\section{Case 1}

An 82-year-old man was referred to OPEN ARCH by his GP following a recent decline in mobility on a background of previous stroke, type 2 diabetes and heart disease. Concerns from the GP included: unreliable family support following missed GP and specialist appointments and medication compliance. The initial CGA was conducted by the EO and geriatrician at his home, revealing that he:

- is living with his adult son who works away;

- has unsteady mobilisation with increasing risk of falling;

- has difficulty toileting at night as dependent for bed transfers;

- is frustrated by reliance on his son for transport, with consequential social isolation and inability to maintain medical appointments; and

- is experiencing difficulty in understanding and following his medication regime.

The following management was instituted:

(1) Negotiation with provider to include transport to medical appointments and a falls alarm within his existing Home Care Package (HCP).

(2) An occupational therapy assessment resulting in minor bathroom modifications and provision of an electric bed and scooter (with funds utilised from his HCP).

(3) Medication review by the geriatrician identifying opportunities for deprescribing and medication optimisation, including changes in the management of osteoporosis to reduce complications from falls.

The outcomes of these interventions resulted in improved independence and quality of life. Specifically, the client attended all scheduled medical appointments, gained an independent form of mobility that enabled him to visit the chemist to fill scripts and joined the local men's shed. At 6-month postintervention, this client has had no falls or hospital admissions.

\section{Alignment of specialist/generalist care}

OPEN ARCH aligns geriatric specialist and generalist perspectives. The GP remains as the central medical decision-maker, with the EO and geriatrician providing specialist advice to the GP regarding the suggested course of treatment and required supports. Sharing of medical records is facilitated through specialist access to GP practice software, with assessment information entered in real-time to the client record, negating the wait time for specialist correspondence and improving the timeliness of interventions. A case conference is held with the GP, EO and geriatrician following the CGA, bringing together assessment information and related recommendations.

\section{Case 2}

An 87-year-old woman was referred to OPEN ARCH with concerns regarding her physical and cognitive decline. The OPEN ARCH geriatrician was able to access both the hospital medical notes and the GP records in real time, informing him that: - during a recent admission, the patient had a full work-up by the treating specialist hospital team, including magnetic resonance imaging and screen bloods, identifying significant cerebrovascular disease; 
- there had been a suggested follow-up plan and changes to her medications, including cessation of high-risk medications; however, the interim discharge summary was not yet available to the GP;

- full blood tests had recently been ordered by the GP; and

- there were three recent emergency department (ED) presentations following periods of confusion.

The GP had concerns about how the client was managing alone at home. The EO was able to confirm at case conference that the house was poorly maintained, and that food was limited. Direct clinical discussion between the GP and geriatrician confirmed that although there appeared to be no acute cause for her decline, there were opportunities to optimise cardiovascular risk management. Following her second OPEN ARCH medical review, a diagnosis of vascular dementia was made. With consent, the EO arranged for an Aged Care Assessment Team (ACAT) assessment through My Aged Care, and home support services, including Meals-On-Wheels, were instigated immediately.

As a result of OPEN ARCH intervention, this client has successfully continued to live at home with the support of a level 2 HCP. Additionally, the client's daughter, who was contemplating the need for residential aged care is confident of her mother's ability to remain living at home with formal supports.

\section{Care coordination and enablement}

The EO assists the client and their family to navigate the complexities of the aged care and healthcare systems and access the supports required to keep them living at home for longer. Additionally, through health and social care education, the EO develops client self-management skills that allow the older person and their carer to manage fluctuations in their complex needs and to access the right care at the right time to avoid unnecessary and preventable hospitalisations.

\section{Case 3}

A 72-year-old aged pensioner was referred to OPEN ARCH with complex chronic conditions. Following CGA, the following issues were identified:

- wheelchair-dependent following recent left below-knee amputation;

- uncontrolled diabetes;

- depression;

- increasing carer frailty; and

- increasing dependence for personal and instrumental activities of daily living.

From an enablement perspective, he was referred to Shortterm Restorative Care (STRC; a multidisciplinary program designed to reverse or slow functional decline and improve wellbeing) (Australian Government Department of Health $2019 b$ ), with the goal of increasing functional independence. A coordinated plan of care was arranged, involving diabetes education and psychology support. The EO assisted both the client and carer to access My Aged Care and provided information on advanced care planning and carer supports. The complexity of this case demanded frequent contact to establish required supports and build self-management capacity, and close GP collaboration for clarification of care plans.
Positive outcomes for this client were determined by a collaborative network of supports that were activated by his EO. Through diabetes education, this client was able to commence self-administration of his medication. This client did have one presentation to the ED in the 6 months post-intervention; however, with a detailed care plan developed in the ED in liaison with his EO, he was able to return home rather than be admitted.

\section{Primary care capacity building}

Through collaboration, OPEN ARCH builds the capacity of GPs to provide best practice aged care for their total cohort of older persons, beyond those patients referred to OPEN ARCH. Additionally, within an Aboriginal Community Controlled Health Organisation (ACCHO), the ability to build capacity for geriatric and health and aged care system knowledge within a culturally appropriate framework is vital.

\section{Case 4}

A 65-year-old Aboriginal woman was referred to OPEN ARCH from a local ACCHO. Prior to the first visit by the EO, contact was made with the ACCHO's health worker to obtain a detailed insight into her health, social and cultural circumstances. A joint visit from the EO and health worker was arranged to ensure that the client was supported appropriately during assessment and care planning. During the episode of OPEN ARCH care, the EO was able to facilitate informal training, including the provision of health and aged care information and resources to the health worker. The health worker gave feedback that she had been able to apply the knowledge around understanding and navigating both healthcare and aged care systems with other elderly clients. The geriatrician formed a collaborative partnership with the GPs within the practice providing ongoing in-service training and information session for all staff on management of diseases of ageing within Aboriginal and Torres Strait Islander communities. Specific medical management support for the client involved cultural assessment of mood and cognition.

\section{Conclusion}

Older persons with complex needs require preventative care and coordinated supports to maintain health and wellbeing and to continue living at home. The GP, and primary care more broadly, play a vital role in establishing continuity of care with the capacity to intervene early in the trajectory of the person's illness both medically and socially. OPEN ARCH is a model that complements the role of the GP, through an integrated and collaborative approach to patient-centred care. Through vertical integration at the primary-secondary interface, OPEN ARCH does not only improve the quality of care for clients, but improves the capacity of primary care to meet the needs of this population. The model of OPEN ARCH can be modified to incorporate a telehealth component of geriatrician assessment and teleconferencing, along with face-to-face local EO support that is applicable to rural and remote locations. OPEN ARCH is currently being evaluated through a randomised controlled trial, economic evaluation and qualitative patient and carer experience study. 


\section{Conflicts of interest}

Edward Strivens, Jennifer Mann, Megan Tait and Gillian Williams are members of the OPEN ARCH service delivery team.

\section{Acknowledgements}

We thank all the clients of OPEN ARCH and our GP partners who participated in the pilot of this new service. Thanks to members of both the OPEN ARCH Research and Steering committees. We also acknowledge and thank the North Queensland Primary Health Network (Out-of-Hours Care program) and the Queensland Health Clinical Excellence Division (Integrated Care Innovation Fund) for their financial support of the OPEN ARCH program (neither organisation was involved in the preparation of this manuscript).

\section{References}

Agency for Clinical Innovation (2014) Building partnerships: a framework for integrating care for older people with complex health needs. New South Wales Department of Health: Sydney, NSW, Australia.

Australian Digital Health Agency (2018) What is My Health Record? Available at https://www.myhealthrecord.gov.au/for-you-your-family/ what-is-my-health-record [Verified 10 September 2019]

Australian Government Department of Health (2019a) Health care homes. Available at https://www1.health.gov.au/internet/main/publishing.nsf/ Content/health-care-homes [Verified 10 September 2019]

Australian Government Department of Health (2019b) Short-term restorative care programme. Available at https://agedcare.health.gov.au/ programs-services/flexible-care/short-term-restorative-care-programme [Verified 10 September 2019]

Australian Institute of Health and Welfare (AIHW) (2014) Australia's health 2014. Cat. no. AUS 178, Australia's health series no. 14. AIHW, Canberra, ACT, Australia.

Beswick AD, Rees K, Dieppe P, Avis S, Gooberman-Hill R, Horwood J, Ebrahim S (2008) Complex interventions to improve physical function and maintain independent living in elderly people: a systematic review and meta-analysis. Lancet 371(9614), 725-735. doi:10.1016/S01406736(08)60342-6

Bird SR, Kurowski W, Dickman K, Kronborg I (2007) Integrated care facilitation for older patients with complex health care needs reduces hospital demand. Australian Health Review 31(3), 351-361. doi:10. 1071/AH070451

Curry N, Ham C (2010) 'Clinical and service integration: the route to improved outcomes.' (The Kings Fund: London, UK)

Davy C, Kite E, Aitken G, Dodd G, Rigney J, Hayes J, Van Emden J (2016) What keeps you strong? A systematic review identifying how primary health-care and aged care services can support the well-being of older Indigenous peoples. Australasian Journal on Ageing 35(2), 90-97. doi:10.1111/ajag.12311

De Carvalho IA, Epping-Jordan J, Pot AM, Kelley E, Toro N, Thiyagarajan JA, Beard JR (2017) Organizing integrated health-care services to meet older people's needs. Bulletin of the World Health Organization 95, 756763. doi:10.2471/BLT.16.187617

Goodwin N (2013) Understanding integrated care; a complex process, a fundamental principle. International Journal of Integrated Care 13, e011-e012. doi:10.5334/ijic.1144
Harvey D, Foster M, Strivens E, Quigley R (2017) Improving care coordination for community dwelling older Australians; a longitudinal qualitative study. Australian Health Review 41, 144-150. doi:10.1071/ AH16054

Katterl R, Anikeeva O, Butler C, Brown L, Smith B, Bywood P (2012) Potentially avoidable hospitalisations in Australia: causes for hospitalisations and primary health care interventions. PHCRIS policy issue review. Primary Health Care Research \& Information Service, Adelaide, SA, Australia.

Leutz WN (1999) Five laws for integrating medical and social services: lessons from the United States and the United Kingdom. The Milbank Quarterly 77(1), 77-110. doi:10.1111/1468-0009.00125

McPake B, Mahal A (2017) Addressing the needs of an ageing population in the health system: the Australian case. Health Systems and Reform 3(3), 236-247. doi:10.1080/23288604.2017.1358796

Mitchell GK, Burridge L, Zhang J, Donald M, Scott IA, Dart J, Jackson CL (2015) Systematic review of integrated models of health care delivered at the primary-secondary interface: how effective is it and what determines effectiveness? Australian Journal of Primary Health 21(4), 391-408. doi:10.1071/PY14172

Productivity Commission (2017) Integrated care, shifting the dial: 5 year productivity review, Supporting Paper Number 4. Productivity Commission, Canberra, ACT, Australia.

Queensland Government (2018) Cairns and Hinterland Hospital and Health Service Clinical Service Plan 2018-2022. State of Queensland, Cairns and Hinterland Hospital and Health Service, Cairns, Qld, Australia.

Smith K, Flicker L, Shadforth G, Carroll E, Ralph N, Atkinson D, Lindeman M, Schaper F, Lautenschlager NT, LoGiudice D (2011) 'Gotta be sit down and worked out together': views of Aboriginal caregivers and service providers on ways to improve dementia care for Aboriginal Australians. Rural and Remote Health Journal 11, 1650.

State of Queensland (2019) Integrated care innovation fund. Available at https://www.health.qld.gov.au/improvement/make-it-happen/integratedcare-innovation-fund [Verified 10 September 2019]

Swierssen H, Duckett S (2016) Chronic failure in primary medical care. Grattan Institute report no. 2016-2. Grattan Institute, Melbourne, Vic., Australia.

Tieman J, Mitchell G, Shelby-James T, Currow D, Fazekas B, O’Doherty L, Hegarty M, Eriksson L, Brown R, Reid-Orr D (2007) Integration, coordination and multidisciplinary care: what can these approaches offer to Australian primary health care? Australian Journal of Primary Health 13(2), 56-65. doi:10.1071/PY07024

Valentijn PP, Schepman SM, Opheij W, Bruijnzeels MA (2013) Understanding integrated care: a comprehensive conceptual framework based on the integrative functions of primary care. International Journal of Integrated Care 13, e010. doi:10.5334/ijic.886

Wodchis WP, Dixon A, Anderson GM, Goodwin N (2015) Integrating care for older people with complex needs; key insights and lessons from a seven-country cross-case analysis. International Journal of Integrated Care 15, e21.

World Health Organization (2016) Integrated care models: an overview. Health Systems Delivery. Programme Division of Health Systems and Public Health, World Health Organization Regional Officer for Europe, Copenhagen, Denmark. 